
\title{
$\begin{array}{ll}\text { Research Square } & \begin{array}{l}\text { Preprints are preliminary reports that have not undergone peer review. } \\ \text { They should not be considered conclusive, used to inform clinical practice, } \\ \text { or referenced by the media as validated information. }\end{array}\end{array}$ \\ Covalently Engineered Nanobody Chimeras for Targeted Membrane Protein Degradation
}

\section{Heng Zhang}

Peking University

\section{Yu Han}

Peking University

\section{Yuanfan Yang \\ Peking University}

Feng Lin

Peking University

Jian Lin

Peking University

Peng Chen ( $\sim$ pengchen@pku.edu.cn )

Peking University https://orcid.org/0000-0002-0402-7417

\section{Article}

Keywords: PROTAC, membrane proteins, therapeutics, biomedical research

Posted Date: March 16th, 2021

DOl: https://doi.org/10.21203/rs.3.rs-306528/v1

License: (c) (1) This work is licensed under a Creative Commons Attribution 4.0 International License. Read Full License 
Covalently Engineered Nanobody Chimeras for Targeted Membrane Protein

Heng Zhang ${ }^{1^{*}}$, Yu Han ${ }^{1,3^{*}}$, Yuanfan Yang ${ }^{1,3}$, Feng Lin ${ }^{1,2,3}$, Jian Lin ${ }^{1,3^{*}}$, Peng R. Chen ${ }^{1,2,3^{*}}$

\section{Affiliations:}

${ }^{1}$ Synthetic and Functional Biomolecules Center, Beijing National Laboratory for Molecular

6 Sciences, College of Chemistry and Molecular Engineering, Peking University, Beijing, China.

$7 \quad{ }^{2}$ Peking-Tsinghua Center for Life Sciences, Academy for Advanced Interdisciplinary Studies, $8 \quad$ Peking University, Beijing 100871, China.

$9{ }^{3}$ Key Laboratory of Bioorganic Chemistry and Molecular Engineering of Ministry of Education, 10 Peking University, Beijing, China.

11 *Corresponding author Email: pengchen@pku.edu.cn (P. R. Chen); linjian@pku.edu.cn (J. Lin) 
Abstract:

Targeted degradation of membrane proteins would afford an attractive and general strategy for treating various diseases that remain difficult with the current PROTAC methodology. We herein report a covalent nanobody-based PROTAC strategy, termed GlueTAC, for targeted membrane protein degradation with high specificity and efficiency. We first designed a MS-based screening platform for rapid development of covalent nanobody (Gluebody) that allowed proximity-enabled ligation with surface antigens on cancer cells. By conjugation with the cellpenetrating peptide and lysosomal-sorting sequence, the resulting GlueTAC chimera exhibited enhanced internalization with high efficacy and sustained eradication of tumor surface antigens such as PD-L1 and EGFR both in vitro and in vivo, which has broad applications in biomedical research and therapeutics.

\section{Main Text:}

Proteolysis targeting chimeras (PROTACs) have rapidly emerged as a powerful strategy for cancer treatment by degradation of oncogenic proteins ${ }^{1,2}$. While the traditional PROTAC methodology has been focused on cytosolic proteins, the recently reported LYTACs ${ }^{3}$ and $\mathrm{AbTACs}^{4}$ offer an attractive approach for membrane protein elimination by targeting cationindependent mannose-6-phosphate receptor (CI-M6PR) or RNF43 with glycopolypeptides and $\mathrm{scFv}$, respectively. Despite their promising results, however, the endocytosis of ternary complexes is difficult due to the large size of the IgG-based antibody scaffolds ${ }^{5}$ as well as the lack of specific receptors or membrane E3 ubiquitin ligase. In addition, the potential immunogenicity of 
34 based membrane protein targeting and degradation platform with a smaller overall size, higher 35 efficiency and wide-applicability without cell-type dependency is highly desirable. smaller size than traditional IgG antibodies with remarkable stability. Although the high penetration capability of Nbs renders them particularly attractive for solid tumors ${ }^{6,7}$, they often have a relatively low binding affinity than full-length antibody, which may cause off-target effects

$\mathrm{V}_{\mathrm{H}} \mathrm{H}$ antibodies or nanobodies (Nbs) are heavy-chain derived antibodies that have a much during endocytosis. Since a stable Nb-Antigen complex is crucial for the endocytosis and degradation processes, we envisioned that covalent binding of the $\mathrm{Nb}$ to its antigen may fulfill this purpose, which also improves its on-target retention in a similar fashion as covalent small molecule drugs $^{8,9}$. Furthermore, the conjugated cell-penetrating peptide (CPP) and lysosome-sorting sequence (LSS) may enhance the internalization and lysosome-mediated degradation of the covalent $\mathrm{Nb}$-antigen complex ${ }^{10,11}$.

Herein, we report the covalent nanobody-based PROTAC strategy, termed GlueTAC, for targeted membrane protein degradation with high specificity and efficiency. Relying on the recently developed proximity enabled covalent binding reaction ${ }^{12-15}$, we created the covalently engineered nanobody chimeras that can irreversibly bind to membrane protein targets via proximity-enabled crosslinking, while the conjugated CPP and LSS allowed the lysosomemediated target degradation (Fig. 1A). We first established a Mass Spectrometry-assisted screening technology (MSSP) for the rapid identification of the crosslinking site between a given antigen $(\mathrm{Ag})$ and a pool of $\mathrm{Nb}$ variants bearing the site-specifically incorporated PrUAA at different locations. As PD-L1 is the major immune checkpoint that is highly expressed in cancer cells for suppressing immune cell responses ${ }^{16-18}$, we coupled this MSSP method with the genetic code expansion (GCE) strategy ${ }^{19}$ to successfully develop the covalent $\mathrm{Nb}$ (Gluebody) for PD-L1, 
and further conjugation with CPP and LSS greatly enhanced the internalization and degradation rate of PD-L1 on cancer cells (Fig. 1B). We further expanded this strategy to another tumor antigen EGFR, which together demonstrated GlueTAC as a general method for efficient and sustained membrane protein degradation in both cancer cells and mice xenograft models.

Development of MSSP for rapid Gluebody screening. As the incorporation sites of the PrUAA in Nbs have decisive impact on the crosslinking efficiency, we developed a MS-based screening platform (MSSP) for rapid identification of Gluebody for an antigen of interest. Our method relies on the site-specific screening of the incorporation sites and the identification of crosslinking peptides by using the tandem Mass Spectrometry (MS) and pLink software ${ }^{20}$ (Fig. 2A). For proof-of-concept, we applied this strategy to the Nb-PD-L1, which could restore T cell cytotoxicity by blocking this major immune checkpoint of cancer cells. According to the crystal structure of Nb-PD-L1/PD-L1 (PDB:5JDR) ${ }^{21}$, we chose the candidate sites for incorporation of PrUAA (FSY) ${ }^{22}$ which are all located in the CDR3 domain, the major binding epitope of Nb-PDL1 (Fig. 2B). The generated pool of Nb-PD-L1 variants containing FSY at selected sites were then incubated with purified PD-L1 protein, followed by SDS-PAGE analysis in which non-covalent protein-protein interactions were disrupted but not covalent Nbs-Ag complexes. To our delight, a new band with the molecular weight corresponding to the Nb-PD-L1/PD-L1 complex was observed and LC-MS/MS analysis indicated that the covalent crosslinking occurred between residues L108FSY on Nb-PD-L1 and His69 on PD-L1 (Fig. 2C, Supplementary Fig. 1).

To verify the covalent binding capacity of Nb-PD-L1 variants at identified sites, the purified FSY-bearing Nb-PD-L1 variants were incubated with PD-L1 and subjected to SDS-PAGE analysis, which showed that Nb-PD-L1-L108FSY, was the only covalent $\mathrm{Nb}$ of the pool that can crosslink 
with PD-L1 (Fig. 2D, Supplementary Fig. 1D). Time-course dependent experiments showed that over $70 \%$ of the crosslinked complex formed within $5 \mathrm{~h}$ at a $2: 1 \mathrm{Nb} / \mathrm{Ag}$ ratio under physiological conditions (Fig. 2E). In addition, we tested another PrUAA (BrC6K $)^{23}$ with a longer and more flexible side-chain, and found that when incorporated at residue Thr110 or Gly113, the resulting variants Nb-PD-L1-T110BrC6K and Nb-PD-L1-G113BrC6K could also crosslink with the PD(Fig. 2G). L1, which was consistent with the longer distance between the $\mathrm{BrC6K}$ incorporation sites and His69 of PD-L1. Of three MSSP-identified covalent Nb-PD-L1 variants with FSY or BrC6K incorporation, Nb-PD-L1-L108FSY exhibited highest crosslinking efficiency, termed as Gluebody-PD-L1 which was utilized in the following experiments.

To further demonstrate that our Gluebody can be covalently attached to the endogenous PDL1 expressed on cell surface, PD-L1/MDA cells were incubated with the TAMRA-labeled Gluebody-PD-L1 followed by washing with stringent buffer that could disrupt the non-covalent nanobody-antigen interaction. Fluorescent imaging showed that only the Gluebody-PD-L1 but not its non-covalent counterpart remained bound to the PD-L1 ${ }^{+}$cells under denaturing conditions (Fig. 2F), indicating the formation of a covalent linkage on cell surface. Western blotting analysis also confirmed the crosslinked conjugate in PD-L1/MDA cells after incubating with Gluebody-PD-L1

Development of GlueTAC for membrane protein degradation. Live-cell imaging showed that self-endocytosis rate of Gluebody-PD-L1 and its complex is relatively low (Fig. 2F), we then aimed to use the CPP-LSS peptide to accelerate the cell entry and degradation process of target proteins. Since the expression of Nbs fused with the positively charged CPP was quite difficult, we decided to conjugate the chemically synthesized functional peptide to the $\mathrm{C}$ terminal of 
103 Gluebody via Sortase A-mediated coupling reaction ${ }^{24}$, which produced the stable and 104 homogeneous chimera with nearly quantitative yield (Fig. 3A, Supplementary Fig. 4). 105 Fluorescence microscopy experiments revealed that the TAMRA-labeled GlueTAC-PD-L1 co106 localized with PD-L1 and lysosomes, indicating that GlueTAC-PD-L1 was able to drive membrane 107 PD-L1 internalization and lysosome trafficking (Fig. 3B, Supplementary Fig. 5).

GlueTAC mediated PD-L1 degradation in vitro and in vivo. We next monitored the degradation of PD-L1 on live cells. The NSCLC cell line HCC827 cells were first treated with 100 nM GlueTAC-PD-L1 for $12 \mathrm{~h}$, with the Gluebody-PD-L1 alone and the non-covalent counterpart NbTAC-PD-L1 used as controls. Western blotting analysis showed that GlueTAC-PD-L1 induced the degradation of PD-L1 with maximal degradation rate of $87 \%$ when compared to NbTAC-PDL1 with a degradation rate of $33 \%$, indicating the essential role of the covalent linkage during the internalization and degradation process. Noteworthy, a clear crosslinked band was observed between Gluebody-PD-L1 and PD-L1, however, a much less degradation occurred, confirming that internalization of Gluebody-PD-L1 alone is not sufficient for degradation (Fig. 3C). In 119 after being treated with GlueTAC-PD-L1 for $12 \mathrm{~h}$ (Fig. 3D). Furthermore, to investigate the degradation mechanism, HCC827 cells were co-incubated with GlueTAC-PD-L1 or controls in the presence and absence of the lysosome inhibitor $\mathrm{NH}_{4} \mathrm{Cl}$. Results showed that degradation of PD-L1 was significantly decreased when adding the lysosome inhibitor (Fig. 3E), demonstrating the dependency on the lysosomal pathway. Finally, to show the generality of GlueTAC mediated protein degradation on typical cancer cell lines, we demonstrated the reduction of $70-80 \%$ PD-L1 
on H460 (lung cancer) (Fig. 3F), U87 (glioblastoma) (Fig. 3G) and PD-L1/MDA (stable cell line)

126 (Fig. 3H) cells after the treatment of GlueTAC-PD-L1.

We went on to demonstrate the therapeutic potential of our GlueTAC for PD-L1 ${ }^{+}$tumors in vivo. PD-L1/MDA cells were engrafted in the immuno-deficient NOG mice to form solid tumors (Fig. 3I). When the tumor size reached $100 \mathrm{~mm}^{3}$, TAMRA labeled Gluebody-PD-L1 was i.v. 130 injected into the NOG mice bearing the PD-L1/MDA derived xenograft. The tumor samples were 131 then dissected, sliced and imaged, and in parallel, digested for western blotting analysis. The 132 results showed that our Gluebody-PD-L1 was covalently bound to tumor cells (Supplementary Fig. 133 6) and was widely spread within the tumor (Fig. 3J), which suggested that the Gluebody-PD-L1 134 retained the excellent permeability of Nbs. We further tested whether GlueTAC could degrade PD$135 \mathrm{~L} 1 \mathrm{in}$ tumor tissues. When the tumor size reached $60 \mathrm{~mm}^{3}$, GlueTAC-PD-L1 and the control constructs were delivered via peri-tumoral injection. After $18 \mathrm{~h}$, the tumors were dissected for immunoblotting analysis which showed apparent degradation of PD-L1 by GlueTAC but not other control constructs (Fig. 3K).

GlueTAC mediated EGFR degradation. To demonstrate the generality of our GlueTAC strategy, we further applied it for the degradation of EGFR, a well-known target antigen for treating cancers ${ }^{25}$. By site-specific incorporation of FSY into Nb-EGFR ${ }^{26}$ followed by MSSP, we found apparent crosslinking between FSY incorporated at Gln116 on Nb-EGFR and His409 of EGFR. The resultant Nb-EGFR-Q116FSY, termed Gluebody-EGFR, was purified, TAMRAlabeled and incubated with the purified EGFR extracellular domain before SDS-PAGE analysis (Supplementary Fig. 7). A covalent complex of Gluebody-EGFR and EGFR was detected, but not for Nb-EGFR, confirming FSY-mediated covalent targeting of EGFR (Supplementary Fig. 8). 
148 Next, to test if Gluebody-based GlueTAC strategy could degrade EGFR on cancer cells, GlueTAC149 EGFR was constructed and incubated with HeLa cells highly expressing EGFR. Western blotting 150 analysis showed that GlueTAC-EGFR demonstrated higher efficiency in decreasing EGFR on 151 HeLa cells over its non-covalent counterpart as well as the non-conjugated Gluebody-EGFR alone. $152 \quad$ (Supplementary Fig. 9).

In summary, conventional therapeutic antibodies inhibit oncogenic signaling from cell154 surface receptors by antagonizing extracellular receptor-ligand interactions, which cannot 155 completely prevent intracellular signaling as many receptors including PD-L1 exhibited ligand156 independent activity. Our covalent nanobody-based GlueTAC strategy thus provides an alternative 157 approach for eliminating surface receptor signaling.

In compare with the recently reported LYTAC and AbTAC technologies, GlueTAC has three unique advantages. Firstly, the irreversible attachment of covalent nanobody (Gluebody) to surface antigen can largely avoid off-target effects during the endocytosis process. This covalent feature also enables Gluebody to improve its half-life through on-target retention. Secondly, the endocytosis is driven by enzymatically conjugated polyarginine peptide which is independent of specific receptor or E3 ligase. This is particularly attractive as the tumors are highly heterogeneous and PD-L1 level in both cancer cells and other immunosuppressive cell types will be increased during the immunotherapy due to the adaptive resistance mechanism of immune escaping ${ }^{27}$. Our cell-type independent degradation strategy would thus allow elimination of PD-L1 regardless of cell types within the tumor microenvironment. Finally, the straightforward protocol for constructing GlueTAC chimeras, in conjunction with its outstanding tissue permeability due to the small overall size, makes it an excellent choice for solid tumor treatment. 
Notably, the MSSP platform offers us a high-throughput strategy capable of simultaneous screening of multiple PrUAAs and incorporation sites, allowing rapid identification of proper incorporation site in nanobodies for generating proximal reactive Gluebody. We envision that, by combining the MSSP strategy and mature nanobody discovery technology, the GlueTAC strategy may offer a general platform for targeted cell-surface protein degradation.

\section{References and Notes:}

1. Sakamoto, K. M. et al. Protacs: chimeric molecules that target proteins to the Skp1-Cullin-F box complex for ubiquitination and degradation. Proc. Natl Acad. Sci. USA 98, 8554-8559 (2001).

2. Bondeson, D. P. et al. Catalytic in vivo protein knockdown by small-molecule PROTACs. Nat. Chem. Biol. 11, 611-617 (2015).

3. Banik, S. M. et al. Lysosome-targeting chimaeras for degradation of extracellular proteins. Nature 584, 291-297 (2020).

4. Cotton, A. D., Nguyen, D. P., Gramespacher, J. A., Seiple, I. B. \& Wells, J. A. Development of Antibody-Based PROTACs for the Degradation of the Cell-Surface Immune Checkpoint Protein PD-L1. J. Am. Chem. Soc. 143, 593-598 (2021).

5. Carter, P. J., Hazuda, D. \& Wells, J. A. Next generation therapeutics. Curr. Opin. Chem. Biol. 17, 317-319 (2013).

6. Rashidian, M. et al. Noninvasive imaging of immune responses. Proc. Natl Acad. Sci. USA 112, 6146-6151 (2015).

7. Fang, T. et al. Nanobody immunostaining for correlated light and electron microscopy with preservation of ultrastructure. Nat. Methods 15, 1029-1032 (2018).

8. Janes, M. R. et al. Targeting KRAS Mutant Cancers with a Covalent G12C-Specific Inhibitor. Cell 172, 578-589 (2018).

9. Tamura, T. et al. Rapid labelling and covalent inhibition of intracellular native proteins using ligand-directed N-acyl-N-alkyl sulfonamide. Nat. Commun. 9, 1870 (2018).

10. Han, Y. et al. Protein labeling approach to improve lysosomal targeting and efficacy of 
antibody-drug conjugates. Org. Biol. Chem. 18, 3193-3194 (2020).

11. Verdurmen, W. P. R., Mazlami, M. \& Pluckthun, A. A quantitative comparison of cytosolic delivery via different protein uptake systems. Sci. Rep. 7, 13194 (2017).

12. Xiang, Z. et al. Adding an unnatural covalent bond to proteins through proximity-enhanced bioreactivity. Nat. Methods 10, 885-888 (2013).

13. Furman, J. L. et al. A genetically encoded aza-Michael acceptor for covalent cross-linking of protein-receptor complexes. J. Am. Chem. Soc. 136, 8411-8417 (2014).

14. Li, Q. et al. Developing Covalent Protein Drugs via Proximity-Enabled Reactive Therapeutics. Cell 182, 85-97 (2020).

15. Mortenson, D. E. et al. "Inverse Drug Discovery" Strategy To Identify Proteins That Are Targeted by Latent Electrophiles As Exemplified by Aryl Fluorosulfates. J. Am. Chem. Soc. 140, 200-210 (2018).

16. Dong, H. et al. Tumor-associated B7-H1 promotes T-cell apoptosis: a potential mechanism of immune evasion. Nat. Med. 8, 793-800 (2002).

17. Ribas, A. \& Wolchok, J. D. Cancer immunotherapy using checkpoint blockade. Science 359, 1350-1355 (2018).

18. Neri, D. Antibody-Cytokine Fusions: Versatile Products for the Modulation of Anticancer Immunity. Cancer Immunol. Res. 7, 348-354 (2019).

19. Chin, J. W. Expanding and reprogramming the genetic code. Nature 550, 53-60 (2017).

20. Chen, Z. L. et al. A high-speed search engine pLink 2 with systematic evaluation for proteomescale identification of cross-linked peptides. Nat. Commun. 10, 3404 (2019).

21. Zhang, F. et al. Structural basis of a novel PD-L1 nanobody for immune checkpoint blockade. Cell Discov. 3, 17004 (2017).

22. Wang, N. et al. Genetically Encoding Fluorosulfate-l-tyrosine To React with Lysine, Histidine, and Tyrosine via SuFEx in Proteins in Vivo. J. Am. Chem. Soc. 140, 4995-4999 (2018).

23. Chen, X. H. et al. Genetically encoding an electrophilic amino acid for protein stapling and covalent binding to native receptors. ACS Chem. Biol. 9, 1956-1961 (2014).

24. Chen, L. et al. Improved variants of SrtA for site-specific conjugation on antibodies and proteins with high efficiency. Sci. Rep. 6, 31899 (2016).

25. Yang, $X$. et al. Targeting the tumor microenvironment with interferon-beta bridges innate and adaptive immune responses. Cancer Cell 25, 37-48 (2014). 
26. Schmitz, K. R., Bagchi, A., Roovers, R. C., van Bergen en Henegouwen, P. M. \& Ferguson, K. M. Structural evaluation of EGFR inhibition mechanisms for nanobodies/VHH domains. Structure 21, 1214-1224 (2013).

27. Taube, J. M. et al. Colocalization of inflammatory response with B7-h1 expression in human melanocytic lesions supports an adaptive resistance mechanism of immune escape. Sci. Transl. Med. 4, 127ra137 (2012).

Acknowledgments: We thank Dr. Yanjun Liu and Ms. Kexin Li for expert technical assistance, as well as Dr. Xinyuan Fan and Dr. Jie Wang for helpful discussions. This work was supported by research grants from the National Key Research and Development Program of China (2016YFA0501500 to P. R. Chen), the National Natural Science Foundation of China (21432002 and 21521003 to P. R. Chen), National Science and Technology Major Projects for "Major New Drugs Innovation and Development” (2018ZX09711003 to J. Lin). P. R. Chen was also supported by Peking-Tsinghua Center for Life Sciences, and the Tencent Foundation through the XPLORER PRIZE.

Author contributions: H. Zhang, Y. Han, J. Lin and P. R. Chen designed the research and conducted most of the experiments. Y. Yang synthesized the FSY. F. Lin conducted some in vitro assays. H. Zhang, Y. Han, J. Lin and P. R. Chen wrote the paper with input from all authors. The experimental data sets used in this study are available as supplementary materials.

Competing interests: The authors declare no competing interests.

Correspondence and requests for materials should be addressed to P. R. Chen and J. Lin. Information. More details are available from the corresponding author upon request. 


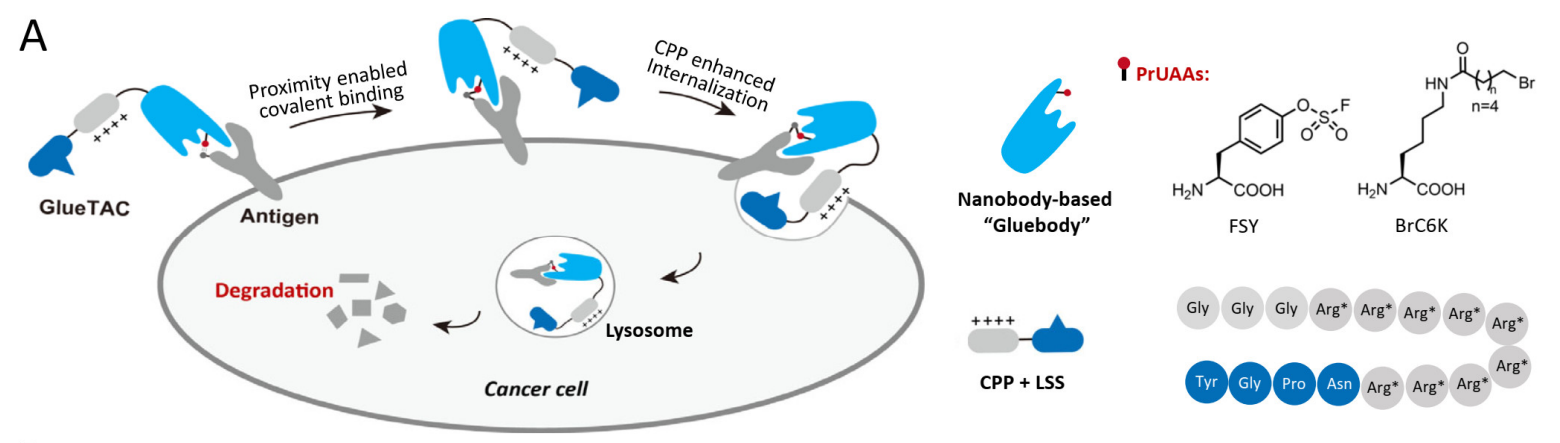

B

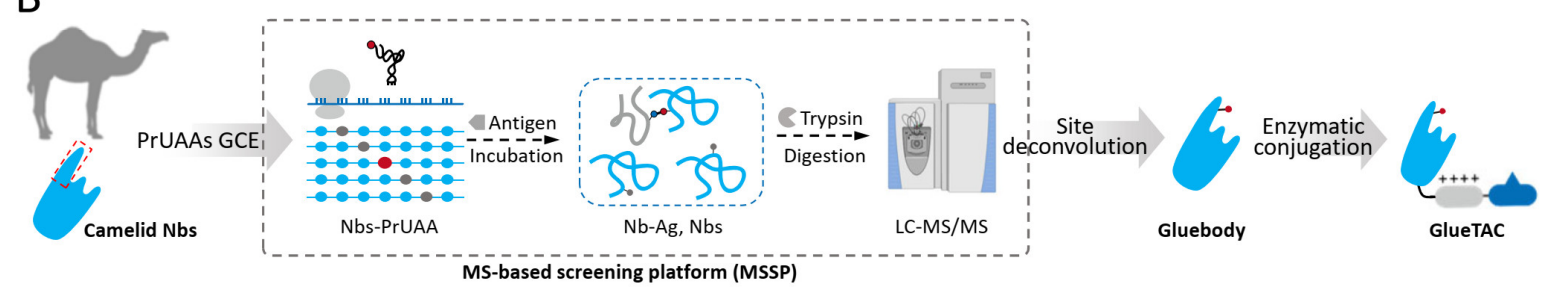
targeted membrane protein degradation. (A) Schematic illustration of the GlueTAC strategy. The nanobody variant containing a site-specifically incorporated proximal reactive uncanonical amino acid (PrUAA, e.g., FSY, BrC6K) can covalently bind to the membrane antigen on tumor cells via proximity-enabled crosslinking. The resulting Gluebody can be further conjugated with the cell-penetrating peptide (CPP) consisting of nine $D$-Arginine and lysosomal-sorting sequence (LSS) consisting of NPGY to generate the final GlueTAC construct with enhanced internalization and degradation of the bound membrane antigen. * means $D$ conformation. (B) Schematic illustration of the pipeline for preparation of GlueTAC. High affinity camelid nanobodies (Nbs) are genetically incorporated with PrUAAs at various sites in the CDR3 region (red frame), resulting a pool of Nbs-PrUAAs. The MS-based screening platform (MSSP) can then be applied to this Nbs pool to identify the covalent variant that exhibited the highest crosslinking efficiency with the antigen. Further enzymatic conjugation of CPP and LSS to this covalent Nb (Gluebody) will produce the desired GlueTAC construct for targeted membrane protein degradation. 
A

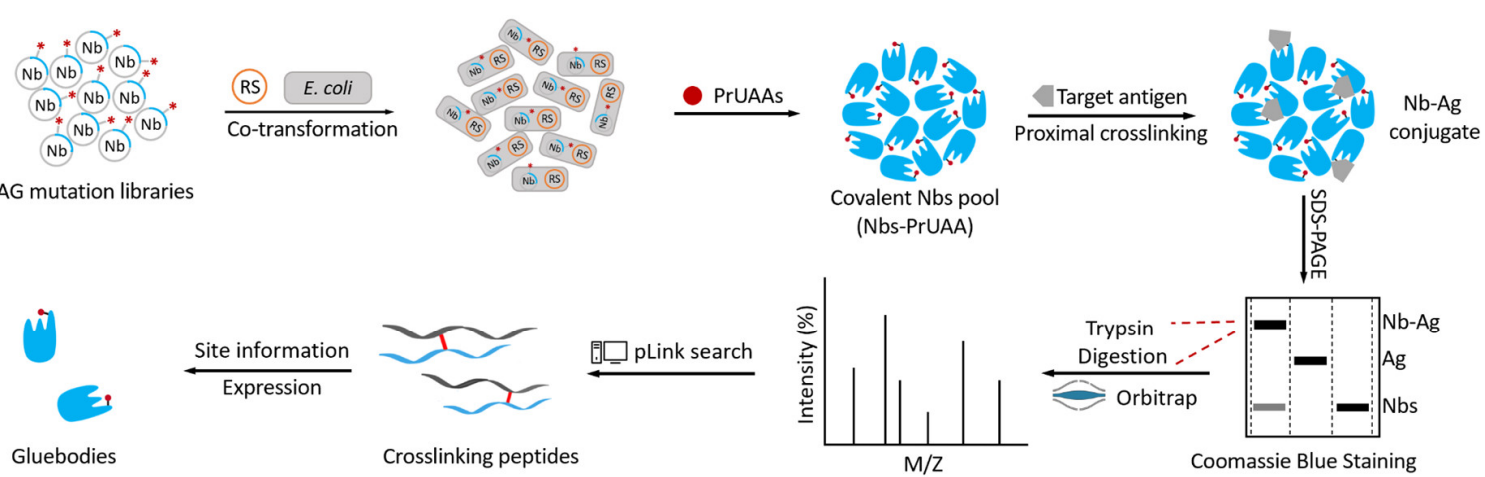

B

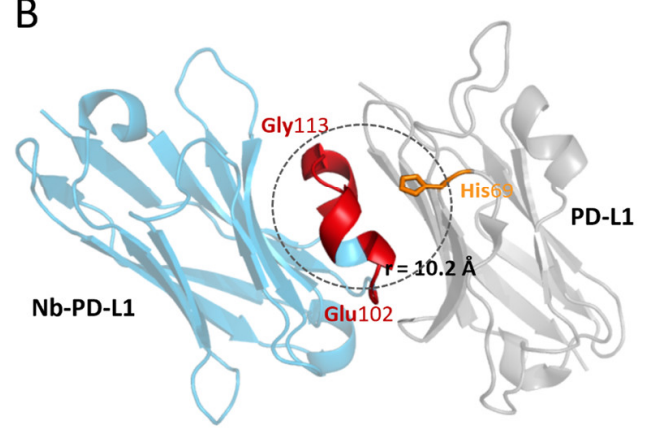

C

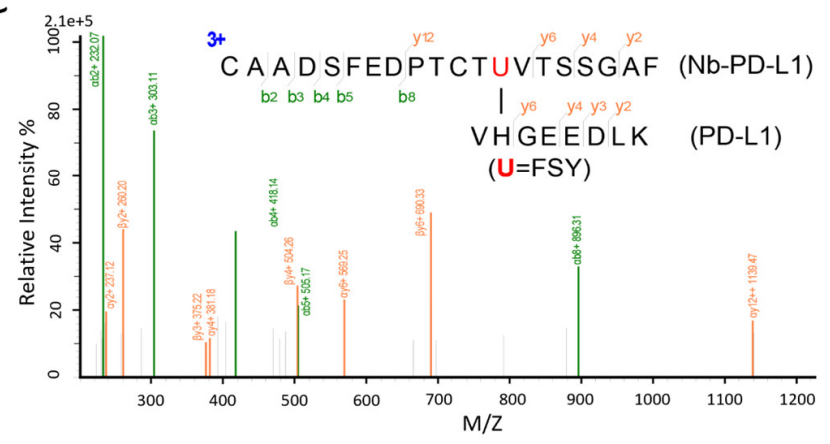

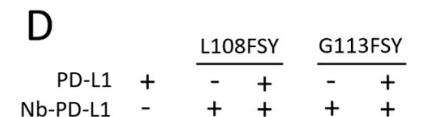
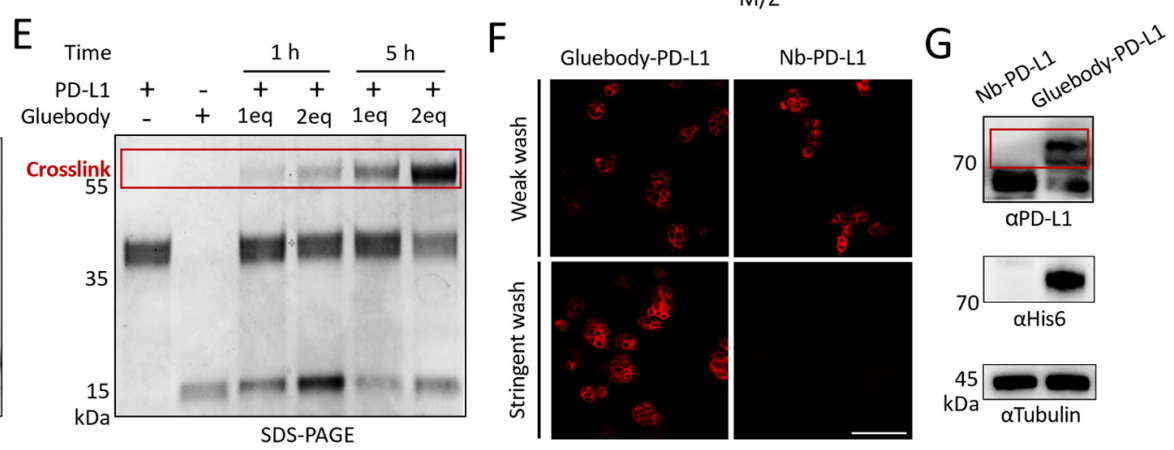

Fig. 2. The MS-based screening platform (MSSP) for Gluebody development and verification. (A) Diagram of the MSSP selection strategy for identifying the covalent nanobody (Gluebody) for an antigen of interest. The selected residues in the CDR3 region of a native nanobody were mutated to amber suppression codon (TAG) to generate the covalent Nbs pool in the presence of both PrUAA and the corresponding aaRS/tRNA pair. Candidate covalent Nbs were incubated with the antigen to initiate in situ, proximity-induced crosslinking, followed by SDS-PAGE analysis and trypsin digestion of the crosslinked bands. The crosslinking peptides can be identified by searching the resulting tandem mass spectrum by pLink software, with the PrUAA incorporation site deconvoluted and the corresponding $\mathrm{Nb}$ variant named as the Gluebody. (B) Structure of PD-L1 in complex with the Nb-PD-L1. The side-chain of His69 on the PD-L1 antigen and the $\alpha$-helix between Glu102 and Gly113 on Nb-PD-L1 are highlighted, which are within 10.2 A distance. PDB: 5jdr. (C) Tandem mass spectrum of the crosslinked Gluebody-PD-L1/PD-L1complex indicated that L108FSYof Nb-PD-L1 reacted with His69 on PD-L1. (D) Verification of the covalent binding of Gluebody-PD-L1 to PD-L1 by SDS-PAGE. Purified proteins were incubated with PD-L1 in PBS buffer at $37^{\circ} \mathrm{C}$ for $5 \mathrm{~h}$ before SDS-PAGE and coomassie staining analysis. Covalent complex 
of Gluebody-PD-L1/PD-L1 was indicated by the red frame. (E) Time-course and dosage study of the reaction between Gluebody-PD-L1 and PD-L1 as verified by SDS-PAGE. (F) Irreversible binding of Gluebody-PD-L1 to the endogenous PD-L1 on PD-L1/MDA cells indicated by fluorescence imaging and $(\mathrm{G})$ western blotting analysis. PD-L1/MDA cells were incubated with TAMRA labeled Gluebody-PD-L1 or Nb-PD-L1 in medium at $37^{\circ} \mathrm{C}$ for $5 \mathrm{~h}$, after which the cells were washed with weak washing buffer or stringent washing buffer (denaturing condition). The crosslinked band was indicated by the red frame. Scale bar $=100 \mu \mathrm{m}$. 

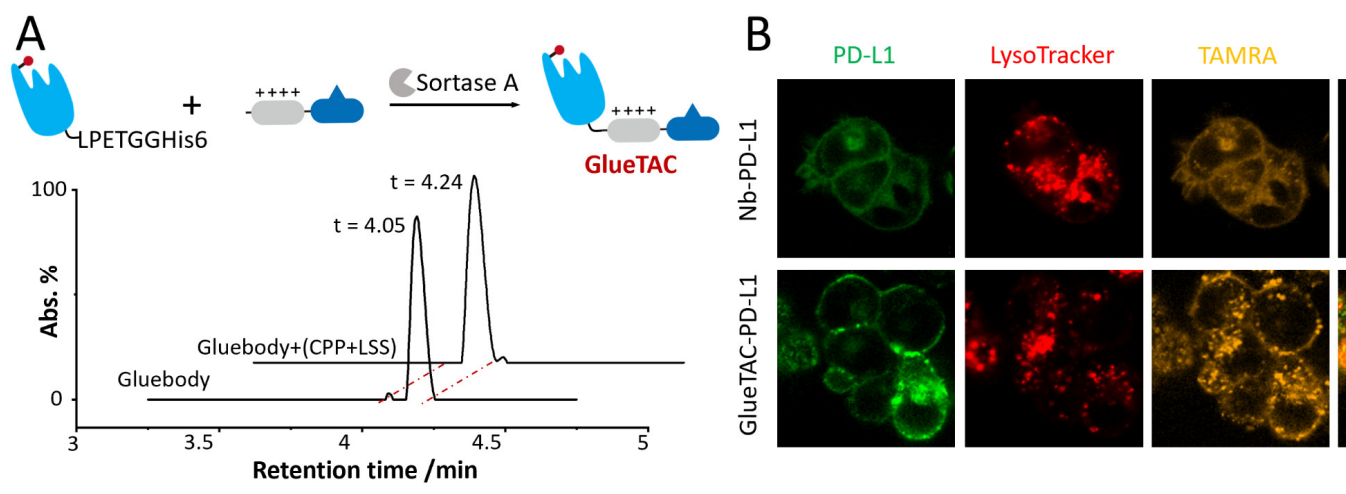

Merged
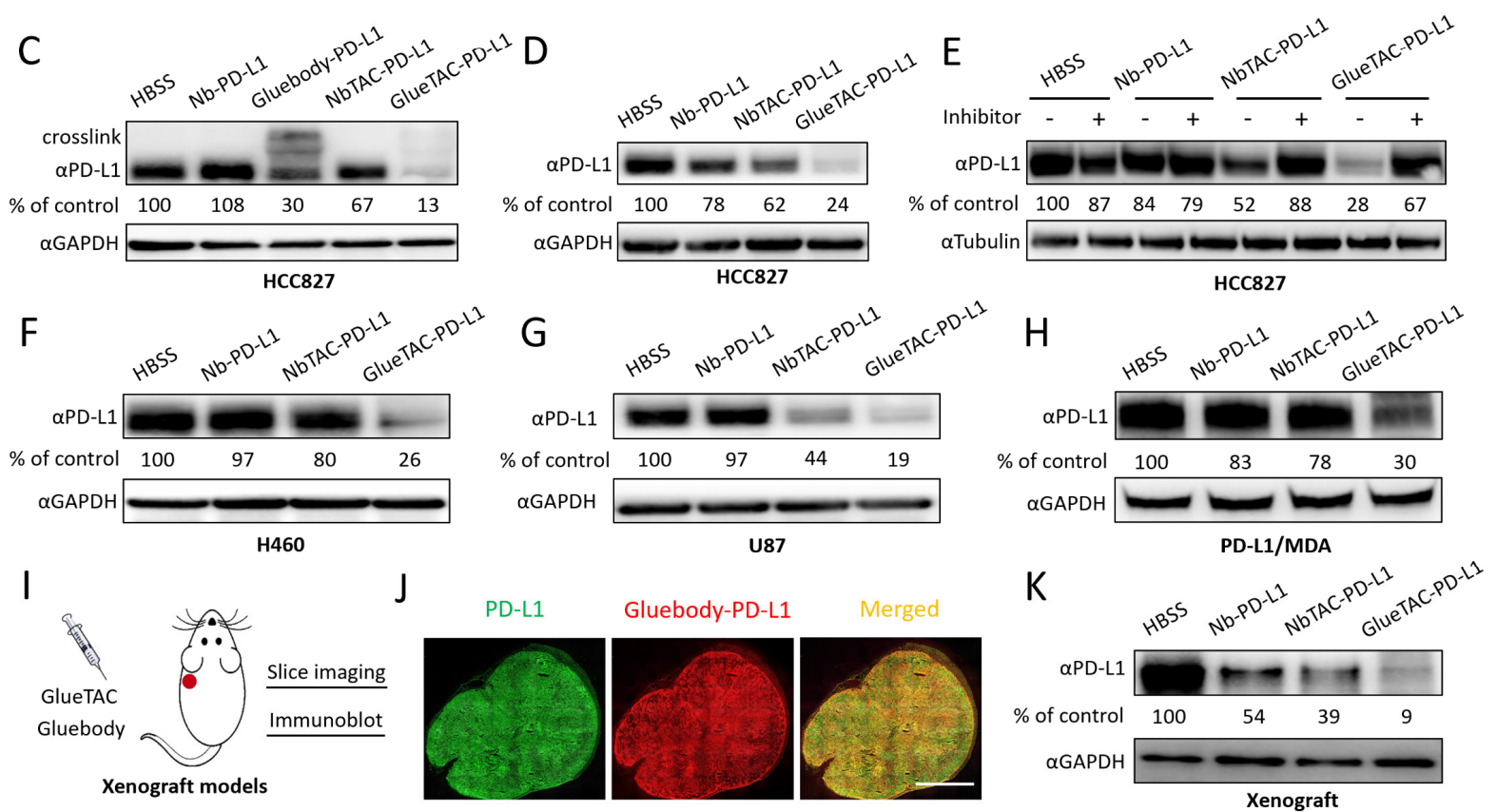

Fig. 3 Targeted degradation of PD-L1 on cancer cells by GlueTAC in vitro and in vivo. (A) Verification of GlueTAC-PD-L1 construction through enzymatic conjugation between the Gluebody-PD-L1 and the CPP-LSS peptide. The functional peptide was added to the C-terminal of Gluebody-PD-L1 through the LPETG tag via Sortase A mediated conjugation and validated by LC-MS analysis. (B) Live-cell confocal microscopy imaging confirms the co-localization of the PD-L1-GFP and TAMRA labeled Nb-PD-L1 or GlueTAC-PD-L1 on tumor cells. The enhanced internalization and lysosomal trafficking of GlueTAC-PD-L1 bound PD-L1-GFP was indicated by LysoTracker Deep Red. Scale bar $=10 \mu \mathrm{m}$. (C) Western blotting analysis confirms PD-L1 degradation mediated by GlueTAC on tumor cells. PD-L1 levels in HCC827 cells after the treatment of GlueTAC-PD-L1 or control constructs in RPMI 1640 supplemented with 10\% FBS at $37{ }^{\circ} \mathrm{C}$ for $12 \mathrm{~h}$. (D) GlueTAC exhibits a sustained degradation effect. Recovery of PD-L1 levels in HCC 827 cells after being cultured with GlueTAC-PD-L1 or controls for $12 \mathrm{~h}$ and a further incubation in fresh medium for another $12 \mathrm{~h}$. (E) PD-L1 levels in HCC827 cells after treatment with GlueTAC-PD-L1 or controls for $12 \mathrm{~h}$ in the presence and absence of lysosome inhibitor (10 $\mathrm{mM} \mathrm{NH}_{4} \mathrm{Cl}$ ). (F) GlueTAC mediates PD-L1 degradation in H460 cells, (G) U87 cells or (H) PDL1/MDA cells for $12 \mathrm{~h}$, respectively. (I) NOG mice bearing PD-L1/MDA derived xenograft tumor 
underarm. (J) Fluorescent images of tissue slice of separated PD-L1 ${ }^{+}$tumors from the xenograft models after i.v. injection with TAMRA-labeled Gluebody-PD-L1 for $4 \mathrm{~h}$. Scale bar $=2 \mathrm{~mm}$. (K) PD-L1 levels in tumors after peri-tumoral injection with GlueTAC-PD-L1 or control constructs 320 for $18 \mathrm{~h}$. The presented data are representative of three independent replicates. 


\section{Figures}

A
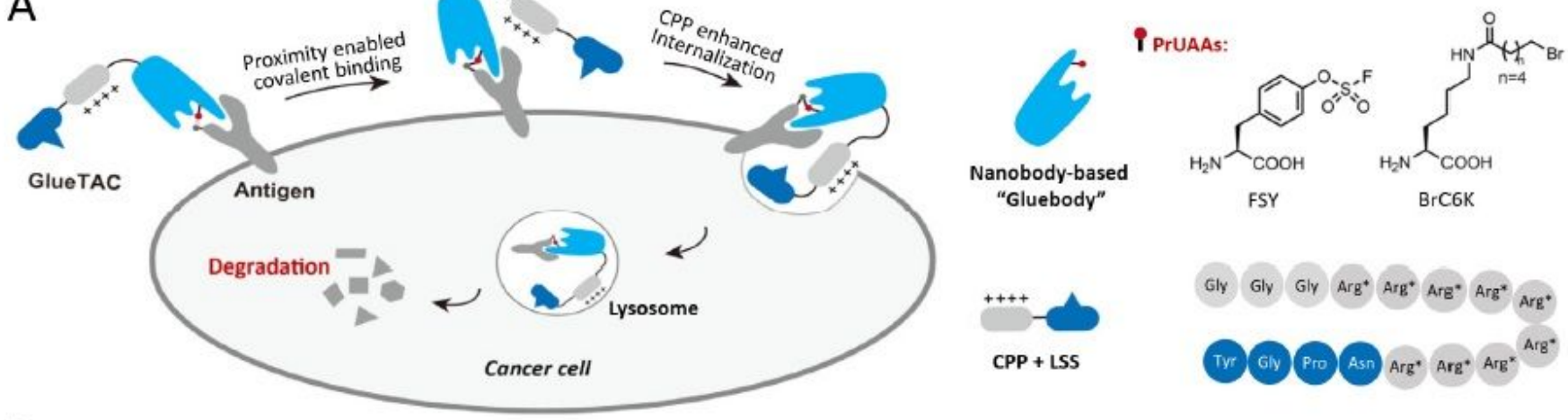

B

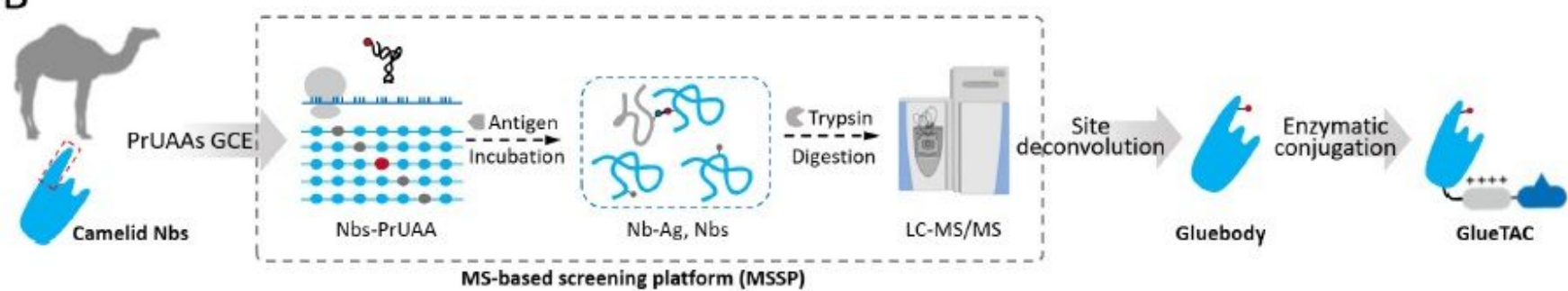

\section{Figure 1}

Design and development of the Gluebody chimera-based GlueTAC strategy for targeted membrane protein degradation. (A) Schematic illustration of the GlueTAC strategy. The nanobody variant containing a site-specifically incorporated proximal reactive uncanonical amino acid (PrUAA, e.g., FSY, BrC6K) can covalently bind to the membrane antigen on tumor cells via proximity-enabled crosslinking. The resulting Gluebody can be further conjugated with the cell-penetrating peptide (CPP) consisting of nine D-Arginine and lysosomal-sorting sequence (LSS) consisting of NPGY to generate the final GlueTAC construct with enhanced internalization and degradation of the bound membrane antigen. * means D conformation. (B) Schematic illustration of the pipeline for preparation of GlueTAC. High affinity camelid nanobodies (Nbs) are genetically incorporated with PrUAAs at various sites in the CDR3 region (red frame), resulting a pool of Nbs-PrUAAs. The MS-based screening platform (MSSP) can then be applied to this Nbs pool to identify the covalent variant that exhibited the highest crosslinking efficiency with the antigen. Further enzymatic conjugation of CPP and LSS to this covalent Nb (Gluebody) will produce the desired GlueTAC construct for targeted membrane protein degradation. 
A

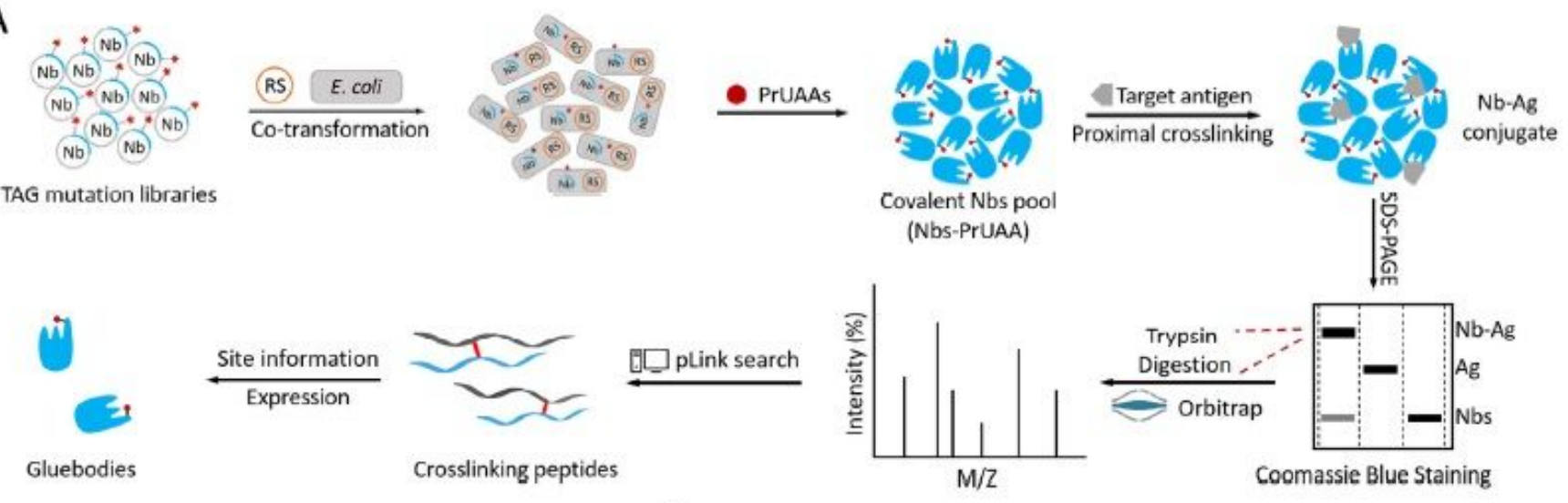

B

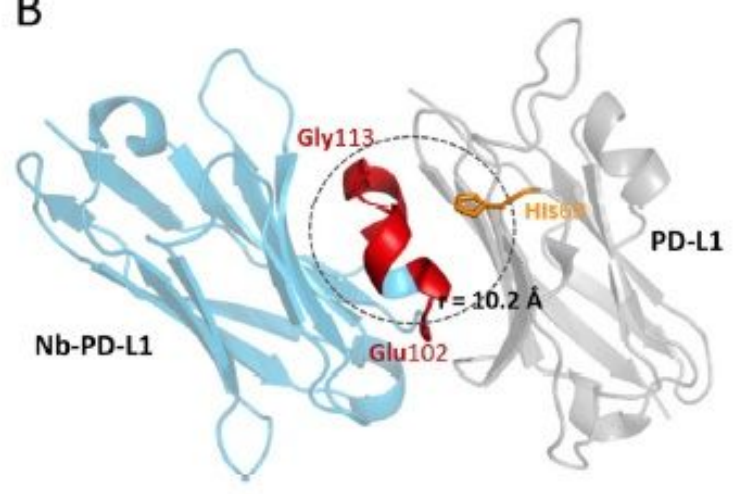

C

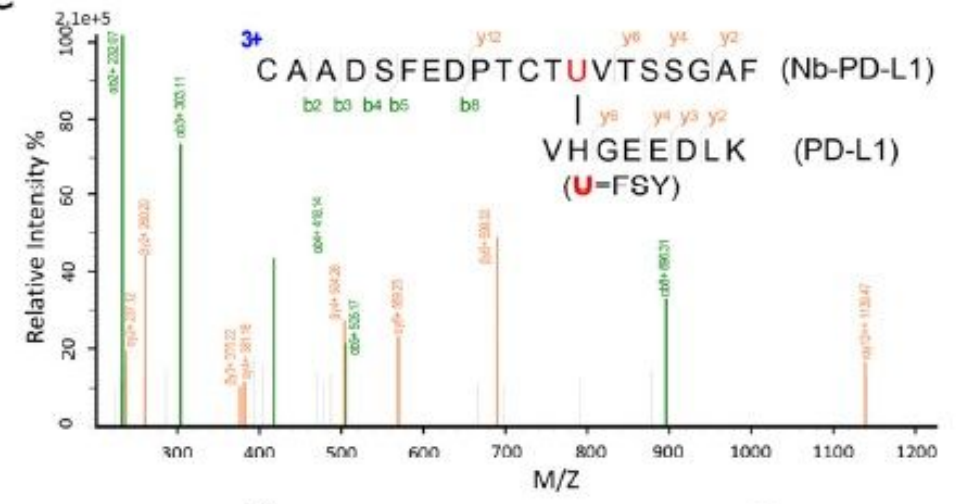

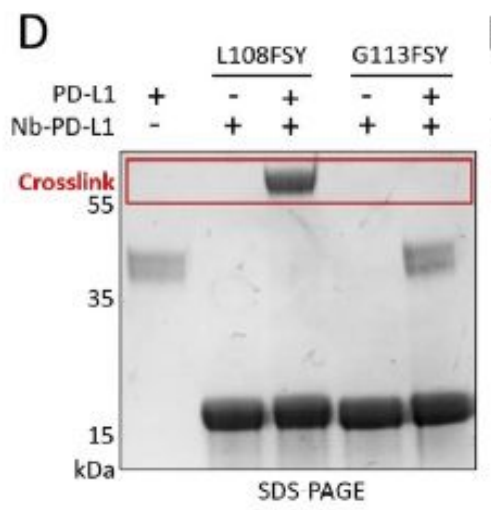
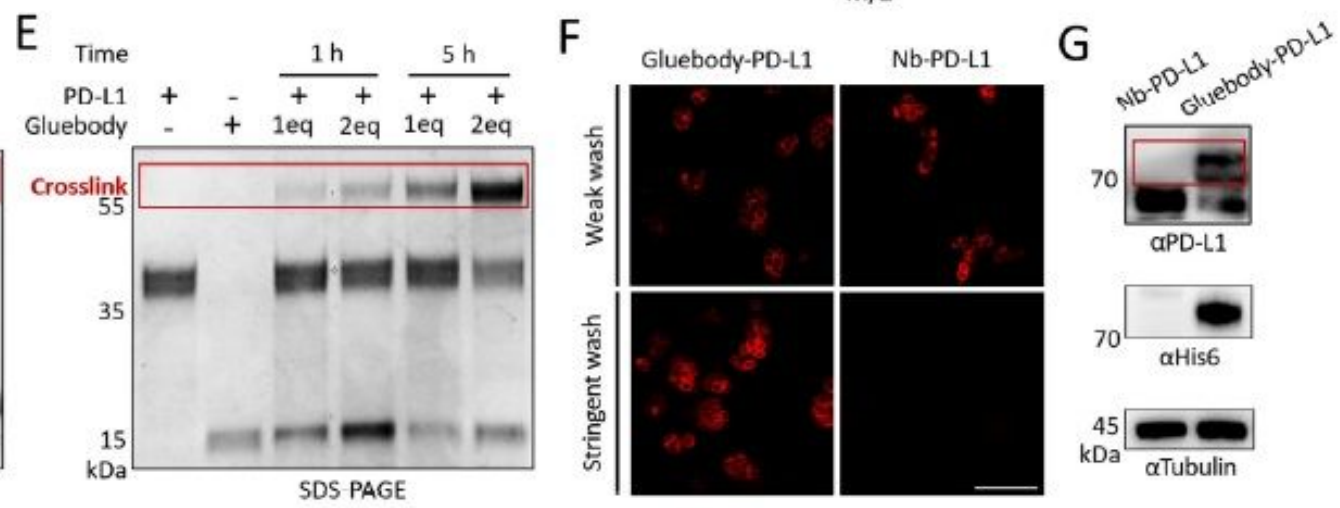

\section{Figure 2}

The MS-based screening platform (MSSP) for Gluebody development and verification. (A) Diagram of the MSSP selection strategy for identifying the covalent nanobody (Gluebody) for an antigen of interest. The selected residues in the CDR3 region of a native nanobody were mutated to amber suppression codon (TAG) to generate the covalent Nbs pool in the presence of both PrUAA and the corresponding aaRS/tRNA pair. Candidate covalent Nbs were incubated with the antigen to initiate in situ, proximity-induced crosslinking, followed by SDS-PAGE analysis and trypsin digestion of the crosslinked bands. The crosslinking peptides can be identified by searching the resulting tandem mass spectrum by pLink software, with the PrUAA incorporation site deconvoluted and the corresponding $\mathrm{Nb}$ variant named as the Gluebody. (B) Structure of PD-L1 in complex with the Nb-PD-L1. The side-chain of His69 on the PD-L1 antigen and the a-helix between Glu102 and Gly113 on Nb-PD-L1 are highlighted, which are within $10.2 \AA$ distance. PDB: 5jdr. (C) Tandem mass spectrum of the crosslinked Gluebody-PD-L1/PD-L1complex 
indicated that L108FSYof Nb-PD-L1 reacted with His69 on PD-L1. (D) Verification of the covalent binding of Gluebody-PD-L1 to PD-L1 by SDS-PAGE. Purified proteins were incubated with PD-L1 in PBS buffer at $37^{\circ} \mathrm{C}$ for $5 \mathrm{~h}$ before SDS-PAGE and coomassie staining analysis. Covalent complex of Gluebody-PDL1/PD-L1 was indicated by the red frame. (E) Time-course and dosage study of the reaction between Gluebody-PD-L1 and PD-L1 as verified by SDS-PAGE. (F) Irreversible binding of Gluebody-PD-L1 to the endogenous PD-L1 on PD-L1/MDA cells indicated by fluorescence imaging and (G) western blotting analysis. PD-L1/MDA cells were incubated with TAMRA labeled Gluebody-PD-L1 or Nb-PD-L1 in medium at $37^{\circ} \mathrm{C}$ for $5 \mathrm{~h}$, after which the cells were washed with weak washing buffer or stringent washing buffer (denaturing condition). The crosslinked band was indicated by the red frame. Scale bar $=100 \mu \mathrm{m}$.
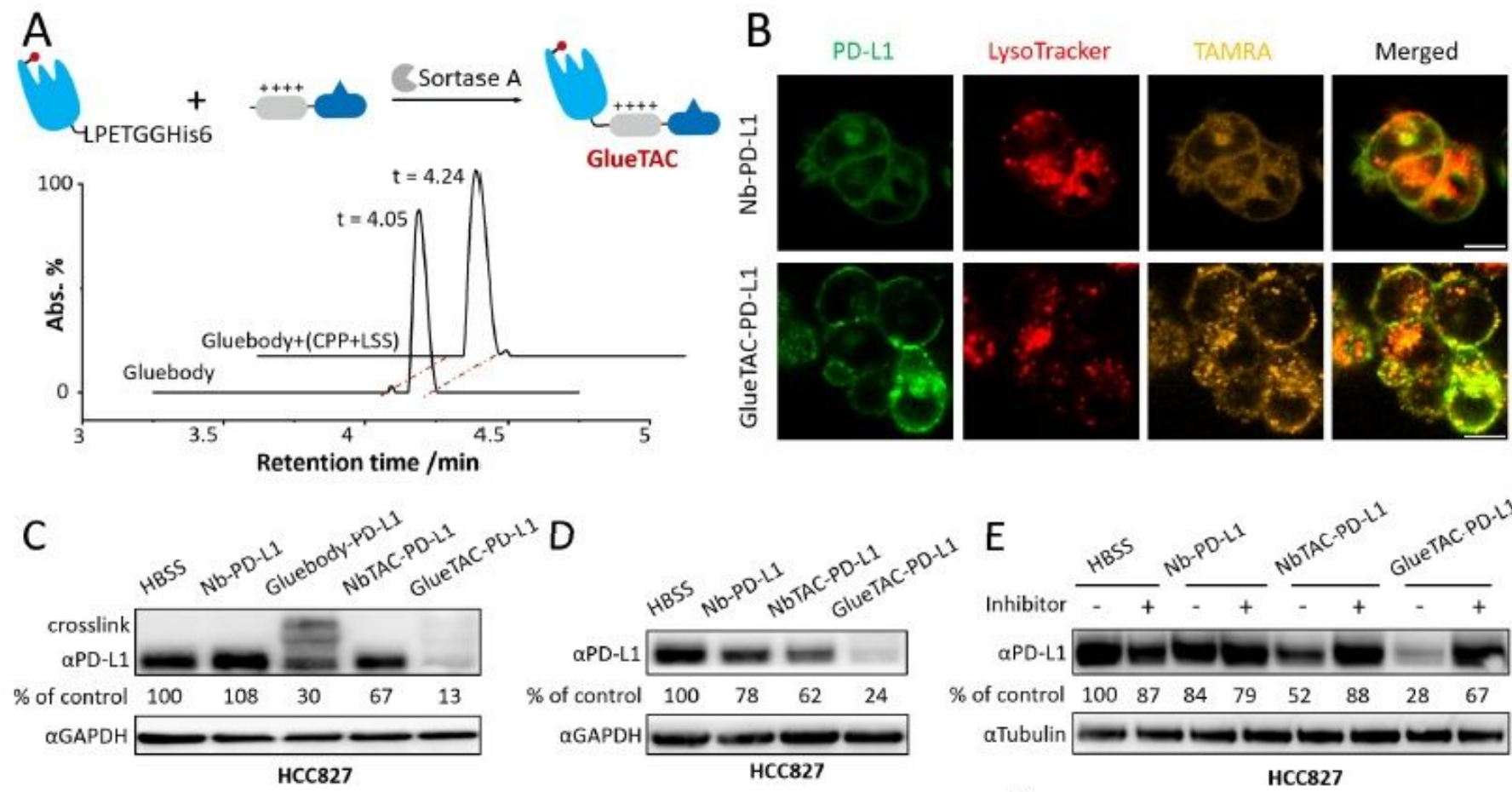
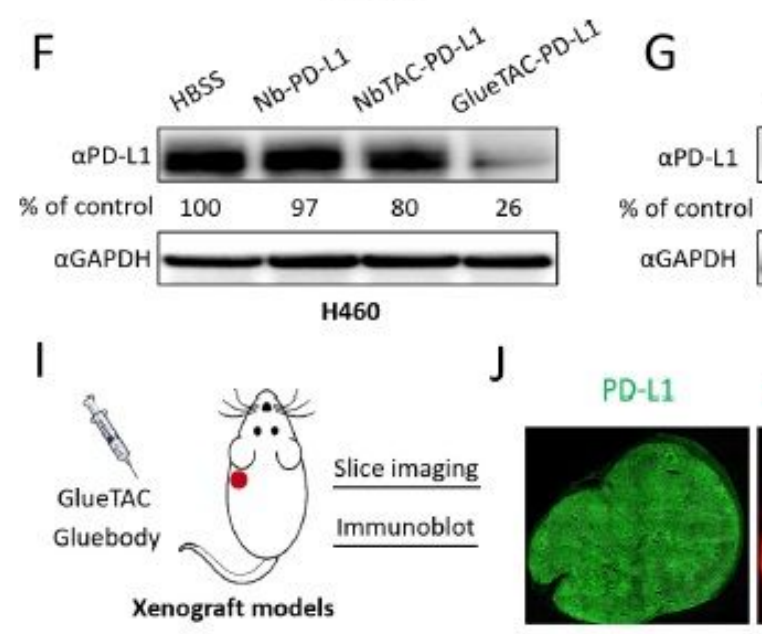
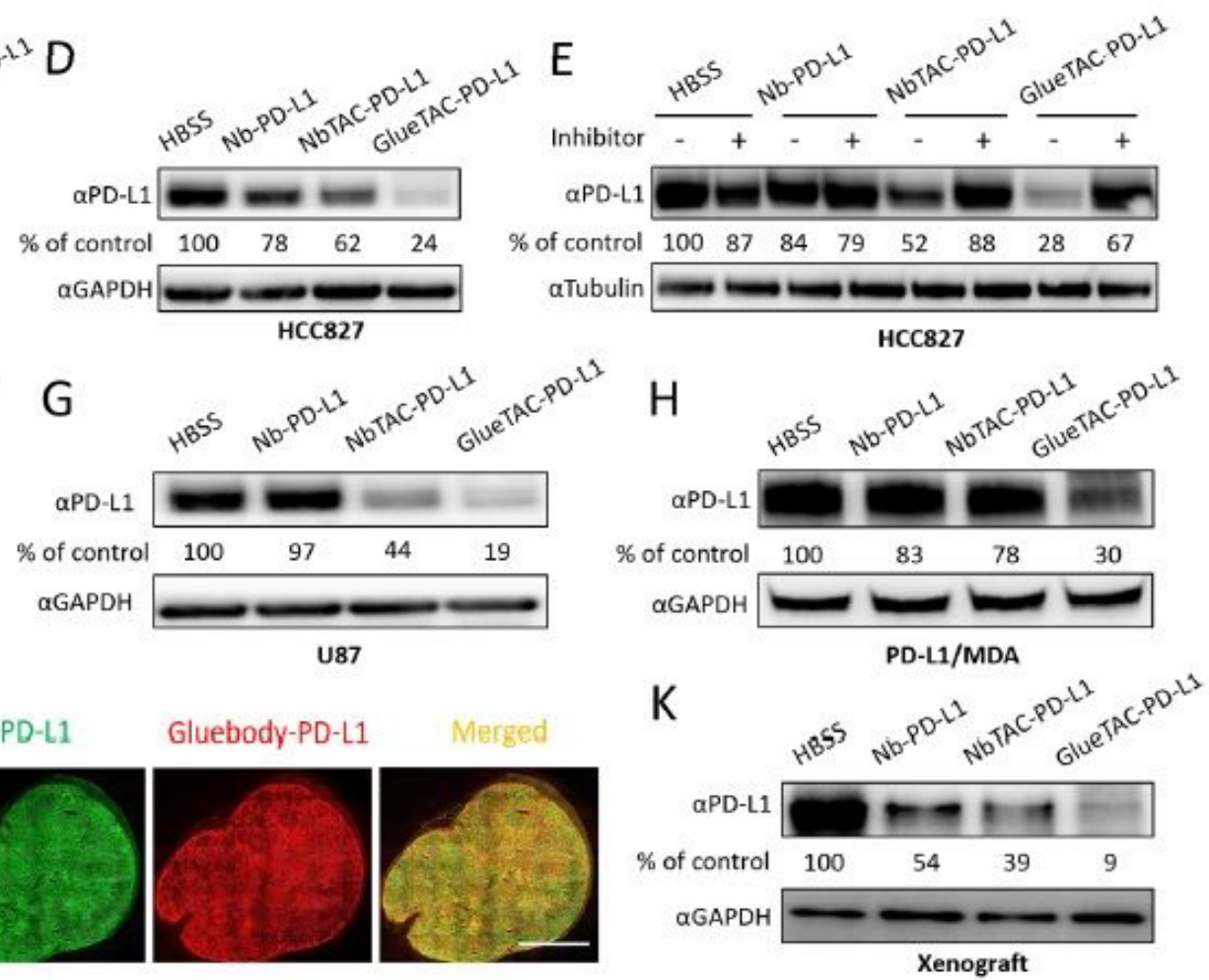

\section{Figure 3}

Targeted degradation of PD-L1 on cancer cells by GlueTAC in vitro and in vivo. (A) Verification of GlueTAC-PD-L1 construction through enzymatic conjugation between the Gluebody-PD-L1 and the CPP- 
LSS peptide. The functional peptide was added to the C-terminal of Gluebody-PD-L1 through the LPETG tag via Sortase A mediated conjugation and validated by LC-MS analysis. (B) Live-cell confocal microscopy imaging confirms the co-localization of the PD-L1-GFP and TAMRA labeled Nb-PD-L1 or GlueTAC-PD-L1 on tumor cells. The enhanced internalization and lysosomal trafficking of GlueTAC-PD-L1 bound PD-L1-GFP was indicated by LysoTracker Deep Red. Scale bar $=10 \mu \mathrm{m}$. (C) Western blotting analysis confirms PD-L1 degradation mediated by GlueTAC on tumor cells. PD-L1 levels in HCC827 cells after the treatment of GlueTAC-PD-L1 or control constructs in RPMI 1640 supplemented with $10 \%$ FBS at $37^{\circ} \mathrm{C}$ for $12 \mathrm{~h}$. (D) GlueTAC exhibits a sustained degradation effect. Recovery of PD-L1 levels in HCC827 cells after being cultured with GlueTAC-PD-L1 or controls for $12 \mathrm{~h}$ and a further incubation in fresh medium for another $12 \mathrm{~h}$. (E) PD-L1 levels in HCC827 cells after treatment with GlueTAC-PD-L1 or controls for $12 \mathrm{~h}$ in the presence and absence of lysosome inhibitor (10 mM NH4Cl). (F) GlueTAC mediates PD-L1 degradation in H460 cells, $(\mathrm{G})$ U87 cells or $(\mathrm{H})$ PD L1/MDA cells for $12 \mathrm{~h}$, respectively. (I) NOG mice bearing PD-L1/MDA derived xenograft tumor underarm. (J) Fluorescent images of tissue slice of separated PD-L1+ tumors from the xenograft models after i.v. injection with TAMRA-labeled GluebodyPD-L1 for $4 \mathrm{~h}$. Scale bar= $2 \mathrm{~mm}$. (K) PD-L1 levels in tumors after peri-tumoral injection with GlueTAC-PD$\mathrm{L} 1$ or control constructs for $18 \mathrm{~h}$. The presented data are representative of three independent replicates.

\section{Supplementary Files}

This is a list of supplementary files associated with this preprint. Click to download.

- Slpdfver2.0.pdf 